\title{
Anticancer activity of sesquiterpenoids extracted from Solanum lyratum via the induction of mitochondria-mediated apoptosis
}

\author{
MIN CHEN $^{1 *}$, JIAN WU $^{2 *}$, XING-XING ZHANG ${ }^{3}$, QIONG WANG $^{4}$, \\ SHI-HAI YAN ${ }^{4}$, HAI-DAN WANG ${ }^{4}$, SHENG-LIN LIU $^{5}$ and XI ZOU ${ }^{5}$ \\ ${ }^{1}$ General Internal Medicine Department; ${ }^{2}$ Central Laboratory; ${ }^{3}$ Digestive Department; \\ ${ }^{4}$ Basic Pharmacology Laboratory; and ${ }^{5}$ Oncology Department, Jiangsu Hospital of Traditional Chinese Medicine, \\ The Affiliated Hospital of Nanjing University of Chinese Medicine, Nanjing, Jiangsu 210029, P.R. China
}

Received July 20, 2015; Accepted October 11, 2016

DOI: $10.3892 / \mathrm{ol} .2016 .5404$

\begin{abstract}
Sesquiterpenoids are a major type of compound found in Solanum lyratum (S. lyratum). The present study aimed to investigate whether sesquiterpenoids from $S$. lyratum demonstrated cytotoxicity against the MCF-7, HCT-8, A-549, SGC-7901 and BEL-7402 cell lines, and the mechanism of solajiangxin $\mathrm{H}$ and lyratol $\mathrm{D}$, which exhibited high cytotoxicity against SGC-7901 cells (half maximal inhibitory concentration, $\mathrm{IC}_{50}=4.8$ and $5.9 \mu \mathrm{g} / \mathrm{ml}$ ), was associated with mitochondria-mediated apoptosis. The results of the Cell Counting Kit- 8 assay indicated that 15 sesquiterpenoids had cytotoxicity against the aforementioned cultured cells. The results of DAPI staining and western blot analysis, used to study the anticancer mechanisms of solajiangxin $\mathrm{H}$ and lyratol D in SGC-7901 cells, suggested that solajiangxin $\mathrm{H}$ and lyratol D induced the apoptosis of SGC-7901 cells significantly $(\mathrm{P}<0.01)$, downregulated the expression of the antiapoptotic proteins $\mathrm{B}$-cell lymphoma $(\mathrm{Bcl})-2$ and survivin, and upregulated the expression of the proapoptotic proteins Bcl-2-like protein 4, second mitochondria-derived activator of caspase, cleaved (c)-caspase-3 and c-caspase-9. The present study therefore demonstrated that 15 sesquiterpenoids from $S$. lyratum exhibited anticancer activity in MCF-7, HCT-8, A-549, SGC-7901 and BEL-7402 cells, and that the anticancer mechanisms of solajiangxin $\mathrm{H}$ and lyratol $\mathrm{D}$ may be associated with
\end{abstract}

Correspondence to: Dr Sheng-Lin Liu or Dr Xi Zou, Oncology Department, Jiangsu Province Hospital of Traditional Chinese Medicine, The Affiliated Hospital of Nanjing University of Chinese Medicine, 155 Hanzhong Road, Nanjing, Jiangsu 210029, P.R. China E-mail: docsl_liu@163.com

E-mail:xizou97@sina.com

${ }^{*}$ Contributed equally

Key words: anticancer activity, sesquiterpenoids, Solanum lyratum, solajiangxin $\mathrm{H}$, lyratol $\mathrm{D}$, apoptosis mitochondria-mediated apoptosis. Additionally, the present study provides evidence in support of the hypothesis that $S$. lyratum may be a promising candidate for the development of novel cancer therapies.

\section{Introduction}

Solanum lyratum (S. lyratum), a common herbal medicine, is widely used in China for the treatment of malaria, icterus, cholecystitis, gonorrhea, rheumatoid arthritis, leucorrhea and cancer (1). Various compounds including saponins, organic acids, terpenoids, polyphenols, flavonoids, sterols, coumarins and polysaccharides have been identified in S. lyratum since the 20th century $(2,3)$. The anticancer activity of $S$. lyratum has received attention, with numerous studies investigating the phenomenon (4-8). Previous studies indicated that water extract from $S$. lyratum induces apoptosis in the human gastric cancer SGC-7901 cell line and HeLa cells, and the mechanism may be associated with changes in the expression level and activity of the apoptotic proteins B-cell lymphoma-extra-large (Bcl-xl), BH3 interacting domain death agonist (Bid), p53, caspase-3 and caspase-9 (4,5). An additional study reported that extracts of $S$. lyratum induced cytotoxicity and apoptosis of the human colon adenocarcinoma Colo 205 cell line, which may be associated with cyclin-dependent kinase 1 (Cdk1), p27, p53, cyclin B1, cyclin E, caspase-3, caspase-8, procaspase-9, B-cell lymphoma-2 (Bcl-2), Bcl-2-like protein 4 (Bax) and cytochrome c activity (6). Additionally, there are studies regarding the cytotoxicity activity of S. lyratum against different cancer cell lines $(7,8)$. However, the aforementioned studies mainly focus on the anticancer activity of the crude extracts of $S$. lyratum, thus the results cannot explain the active anticancer constituent of $S$. lyratum. Thus, an investigation into the anticancer activity and mechanism of the monomeric compounds isolated from $S$. lyratum is required.

Therefore, the presented study explored the anticancer activity and mechanism of the sesquiterpenoids isolated from S. lyratum, which may be useful for the interpretation of the anticancer effect of S. lyratum and the development of novel therapies from this plant. 


\section{Materials and methods}

Plant material. S. lyratum Thunb was obtained from Tongrentang Pharmaceutical Group (Beijing, China) in 2013 and a voucher specimen (voucher no. YP20130913) was stored in Nanjing University of Chinese Medicine (Nanjing, China) for future reference.

Chemicals and reagents. Analytical grade chloroform, ethyl acetate, n-butyl alcohol, cyclohexane, acetone, methanol and silica-gel were obtained from Qingdao Haiyang Chemical Co., Ltd. (Qingdao, China) and Sephadex LH-20 and preparative thin layer chromatography (TLC) were obtained from H\&E Co., Ltd. (Beijing, China). Fetal bovine serum (FBS) and RPMI-1640 media were purchased from Invitrogen (Thermo Fisher Scientific, Inc., Waltham, MA, USA). The Cell Counting Kit (CCK)-8 and DAPI were purchased from Beyotime Institute of Biotechnology (Shanghai, China). Primary antibodies against Bcl-2 (cat. no. AB112; 1:1,000), survivin (cat. no. AS792; 1:1,000), Bax (cat. no. AB026; 1:500), cleaved-caspase (c-caspase)-3 (cat. no. AC033; $1: 1,000), \beta$-actin (cat. no. AF0003; $1: 1,000$ ) and horseradish peroxidase (HRP)-conjugated secondary antibody (cat. no. A0192; 1:1,000) were purchased from Beyotime Institute of Biotechnology (Shanghai, China), while antibodies against second mitochondria-derived activator of caspase (Smac; cat no. $15108 ; 1: 1,000)$ and c-caspase-9 (cat. no. 9501; 1:1,000) were purchased from Cell Signaling Technology (Beverly, MA, USA). BeyoECL Plus reagent was purchased from Beyotime Institute of Biotechnology.

Extraction and isolation. The air-dried whole plant (60 kg) of S. lyratum was finely cut using scissors and extracted by refluxing 4 times with 1201 ethyl alcohol. After filtration, the residues were used for the next extraction. The ethyl alcohol solvent was evaporated under reduced pressure to produce a crude extract $(4.01)$, which was then suspended in water and successfully partitioned into chloroform, ethyl acetate and n-butyl alcohol. The chloroform fraction (238.6 g) was subject to column chromatography (CC) over silica gel (100-200 mesh) and eluted with cyclohexane-acetone (95:5-50:50) to produce 8 fractions. Fraction $3(8.2 \mathrm{~g})$ was separated using $\mathrm{CC}$ over silica gel (cyclohexane-ethyl acetate) to produce compound $1(58 \mathrm{mg})$ and a mixture, this was additionally isolated by preparative thin layer chromatography (TLC; chloroform-ethyl acetate) and purified on Sephadex LH-20 (chloroform-methanol) producing compounds 2 (123 mg) and 3 (73 mg). Fraction 4 (25.4 g) was isolated by CC over silica gel (cyclohexane-ethyl acetate) and preparative TLC (chloroform-acetone) to provide compounds 4 (186 mg), 5 (92 $\mathrm{mg})$ and $6(57 \mathrm{mg})$. Fractions $5(9.1 \mathrm{~g})$ and $6(8.4 \mathrm{~g})$ were separated by CC over silica gel (petroleum ether-acetone) and preparative TLC (cyclohexane-acetone), and purified on Sephadex LH-20 (chloroform-methanol) to provide compounds 7 (93 mg), 8 (51 mg), 9 (105 mg), 10 (108 mg), 11 (61 mg) and $12(72 \mathrm{mg})$. Fractions $2(3.8 \mathrm{~g})$ and $8(19.2 \mathrm{~g})$ were isolated by reversed-phase CC over silica gel (methanol-water) and purified on Sephadex LH-20 (chloroform-methanol) to produce compounds $13(87 \mathrm{mg}), 14(73 \mathrm{mg})$ and $15(68 \mathrm{mg})$. A total of 15 compounds were identified by nuclear magnetic resonance
(NMR) data and compared with the existing literature. The purity of the compounds was verified by high performance liquid chromatography (HPLC). The HPLC analysis was carried out on LC-20AT (Shimadzu, Kyoto, Japan), equipped with an auto sampler, a binary solvent delivery pump and a diode array detector, using Empower 2 software (Waters, Milford, MA, USA). The chromatographic separation was carried out on an Ultimate AQ $C_{18}(4.6 \times 250 \mathrm{~mm}, 5 \mu \mathrm{m}$; Welch, Shanghai, China) at $35^{\circ} \mathrm{C}$. The mobile phase was composed of acetonitrile and water. The injection volume and the flow rate were $10 \mu \mathrm{l}$ and $1.0 \mathrm{ml} / \mathrm{min}$, respectively.

Cell culture. The MCF-7, HCT-8, A-549, SGC-7901 and BEL-7402 cells were purchased from the American Type Culture Collection (Manassas, VA, USA). In total, 5 types of cell were separately cultured in RPMI-1640 medium including $10 \% \mathrm{FBS}, 100 \mathrm{U} / \mathrm{ml}$ penicillin and $100 \mathrm{U} / \mathrm{ml}$ streptomycin at $37^{\circ} \mathrm{C}$ in $5 \% \mathrm{CO}_{2} / 95 \%$ air.

CCK-8 assay. A CCK-8 assay was used to investigate the cytotoxicity of the 15 sesquiterpenoids against cultured MCF-7, HCT-8, A-549, SGC-7901 and BEL-7402 cells and the $\mathrm{IC}_{50}$ value, defined as the concentration that led to a $50 \%$ decrease in the number of cells, was used to evaluate their cytotoxicity. Cells were seeded at a density of $2 \times 10^{4} /$ well into 96 -well plates with RPMI-1640 medium. Subsequent to $24 \mathrm{~h}$ incubation, the cells were treated with different concentrations of fractions and compounds. A total of $48 \mathrm{~h}$ following treatment, CCK-8 solution was added and the cells were cultured at $37^{\circ} \mathrm{C}$ in $5 \%$ $\mathrm{CO}_{2} / 95 \%$ air for $3 \mathrm{~h}$. The optical density was then determined at $450 \mathrm{~nm}$ with a microplate reader (Model 680; Bio-Rad Laboratories, Inc., Hercules, CA, USA).

Apoptosis assay. According to the CCK-8 assay results of the 15 sesquiterpenoids, solajiangxin $\mathrm{H}$ and lyratol $\mathrm{D}$, which had high cytotoxicity against SGC-7901 cells, $\mathrm{IC}_{50}=4.8$ and $5.9 \mu \mathrm{g} / \mathrm{ml}$, respectively, were selected to study their anticancer activities and mechanisms. Following treatment with solajiangxin $\mathrm{H}$ and lyratol D at doses of 0 (control), 2 or $4 \mu \mathrm{g} / \mathrm{ml}$ for $48 \mathrm{~h}$, the SGC-7901 cells were washed 3 times with PBS. The washed SGC-7901 cells were resuspended in staining buffer and stained with DAPI according to the manufacturer's protocol. Images were then captured of the SGC-7901 cells using a fluorescence microscope (BX51; Olympus, Tokyo, Japan).

Western blot assay. Subsequent to treatment with solajiangxin $\mathrm{H}$ and lyratol $\mathrm{D}$ at concentrations of 0 (control), 2, 4 and $8 \mu \mathrm{g} / \mathrm{ml}$ for $48 \mathrm{~h}$, respectively, the SGC-7901 cells were collected and their total proteins were extracted using cell lysis buffer, ultrasound and centrifugation at $12,000 \times \mathrm{g}$ for $15 \mathrm{~min}$ at $4^{\circ} \mathrm{C}$. The concentration of total proteins was determined using an enhanced Bicinchoninic Acid assay kit (Beyotime, Haimen, China) according to the manufacturer's protocol. Subsequently, $\sim 35 \mu \mathrm{g}$ total protein was separated by SDS-PAGE, transferred onto polyvinylidene fluoride membranes and incubated with the primary antibodies. The protein was then incubated with HRP-conjugated secondary antibody, and the anti- and proapoptotic proteins were detected immediately subsequent to incubation using BeyoECL Plus reagent. In addition, 
antibody directed against $\beta$-actin was used to assess the level of protein loading.

Statistical analysis. All data were presented as the mean \pm standard deviation. A one-way analysis of variance using SPSS 21.0 (IBM SPSS, Armonk, NY, USA) determined whether the results showed statistical significance between groups, and $\mathrm{P}<0.05$ was considered to indicate a statistically significant difference.

\section{Results}

Sesquiterpenoids isolated from S. lyratum. A total of 15 sesquiterpenoids, including compounds 1 (solajiangxin I, $\mathrm{C}_{18} \mathrm{H}_{28} \mathrm{O}_{3}$ ), 2 (7-hydroxylsolajiangxin $\mathrm{I}, \mathrm{C}_{18} \mathrm{H}_{28} \mathrm{O}_{4}$ ), 3 (solajiangxin $\mathrm{H}$, $\mathrm{C}_{18} \mathrm{H}_{28} \mathrm{O}_{4}$ ), 4 (lyratol $\mathrm{D}, \mathrm{C}_{15} \mathrm{H}_{20} \mathrm{O}_{3}$ ), 5 (dehydrovomifoliol, $\mathrm{C}_{13} \mathrm{H}_{18} \mathrm{O}_{3}$ ), 6 (blumenol $\mathrm{A}, \mathrm{C}_{13} \mathrm{H}_{20} \mathrm{O}_{3}$ ), 7 (solajiangxin $\mathrm{A}$, $\mathrm{C}_{15} \mathrm{H}_{24} \mathrm{O}_{4}$ ), 8 (solajiangxin $\mathrm{B}, \mathrm{C}_{15} \mathrm{H}_{18} \mathrm{O}_{4}$ ), 9 (solajiangxin $\mathrm{C}$, $\mathrm{C}_{15} \mathrm{H}_{18} \mathrm{O}_{3}$ ), 10 (solajiangxin $\mathrm{D}, \mathrm{C}_{15} \mathrm{H}_{24} \mathrm{O}_{4}$ ), 11 (solajiangxin $\mathrm{E}$, $\mathrm{C}_{18} \mathrm{H}_{28} \mathrm{O}_{3}$ ), 12 (2-hydroxysolajiangxin $\mathrm{E}, \mathrm{C}_{18} \mathrm{H}_{28} \mathrm{O}_{4}$ ), 13 (lyratol $\mathrm{A}, \mathrm{C}_{15} \mathrm{H}_{24} \mathrm{O}_{3}$ ), 14 (lyratol $\mathrm{B}, \mathrm{C}_{15} \mathrm{H}_{24} \mathrm{O}_{3}$ ) and 15 (lyratol $\mathrm{C}$, $\mathrm{C}_{15} \mathrm{H}_{26} \mathrm{O}_{4}$ ) were successfully isolated from $\mathrm{S}$. lyratum, and their chemical structures are shown in Fig. 1. In addition, the NMR data are as follows:

Compound 1 (solajiangxin $\mathrm{I}, \mathrm{C}_{18} \mathrm{H}_{28} \mathrm{O}_{3}$ ) presented as a colorless viscous oil; purity: 97.3\%; ${ }^{1} \mathrm{H}-\mathrm{NMR}(400 \mathrm{MHz}$,

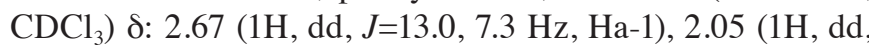
$J=13.0,11.6 \mathrm{~Hz}, \mathrm{Hb}-1), 2.17$ (1H, m, H-2), 1.80 (1H, m, Ha-3), 2.02 (1H, m, Hb-3), 1.86 (1H, m, Ha-4), 1.97 (1H, m, Hb-4), 5.98 (1H, s, H-7), 2.68 (1H, dd, $J=16.8,4.8$ Hz, Ha-9), 2.61 (1H, dd, $J=16.8,4.3 \mathrm{~Hz}, \mathrm{Hb}-9), 2.28(1 \mathrm{H}, \mathrm{m}, \mathrm{H}-10), 3.61(1 \mathrm{H}$, d, J=8.7, Ha-12), 3.74 (1H, d, J=8.7, Hb-12), 1.31 (3H, s, H-13), 1.90 (3H, s, H-14), 1.13 (3H, d, J=7.1, H-15), 1.50 (3H, s, H-2'), 1.49 (3H, s, H-3'); ${ }^{13} \mathrm{C}-\mathrm{NMR}\left(100 \mathrm{MHz}, \mathrm{CDCl}_{3}\right)$ $\delta: 35.5(\mathrm{C}-1)$, 46.3 (C-2), 28.1 (C-3), 33.7 (C-4), 50.6 (C-5), 165.6 (C-6), 124.6 (C-7), 197.6 (C-8), 43.6 (C-9), 39.8 (C-10), 82.1 (C-11), 72.9 (C-12), 24.7 (C-13), 21.6 (C-14), 16.5 (C-15), 109.6 (C-1'), 26.2 (C-2'), 27.7 (C-3') (9).

Compound 2 (7-hydroxylsolajiangxin I, $\mathrm{C}_{18} \mathrm{H}_{28} \mathrm{O}_{4}$ ) presented as a colorless viscous oil; purity: $98.1 \%$; ${ }^{1} \mathrm{H}-\mathrm{NMR}$

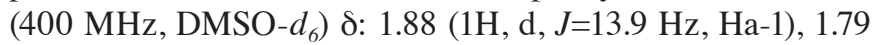
$(1 \mathrm{H}, \mathrm{d}, J=13.9 \mathrm{~Hz}, \mathrm{Hb}-1), 1.56(1 \mathrm{H}, \mathrm{m}, \mathrm{Hb}-3), 1.73(1 \mathrm{H}, \mathrm{m}$, Ha-3), 1.84 (1H, m, Ha-4), $1.98(1 \mathrm{H}, \mathrm{m}, \mathrm{Hb}-4), 5.66(1 \mathrm{H}, \mathrm{s}$, H-7), 2.78 (1H, dd, $J=16.2,5.0 \mathrm{~Hz}, \mathrm{Ha}-9), 2.21$ (1H, dd, $J=16.2$, $4.1 \mathrm{~Hz}, \mathrm{Hb}-9), 2.46$ (1H, m, H-10), 3.81 (1H, d, J=8.5, Ha-12), $4.13(1 \mathrm{H}, \mathrm{d}, J=8.5, \mathrm{Hb}-12), 1.51$ (3H, s, H-13), 1.86 (3H, s, H-14), $1.13(3 \mathrm{H}, \mathrm{d}, \mathrm{J}=7.2, \mathrm{H}-15), 1.20$ (3H, s, H-2'), 1.39 (3H, s, H-3'), 4.67 (1H, s, C2-OH); ${ }^{13} \mathrm{C}-\mathrm{NMR}\left(100 \mathrm{MHz}, \mathrm{DMSO}-d_{6}\right) \delta: 43.2$ (C-1), 84.1 (C-2), 36.6 (C-3), 33.8 (C-4), 50.6 (C-5), 166.4 (C-6), 124.8 (C-7), 198.9 (C-8), 43.1 (C-9), 40.3 (C-10), 85.5 (C-11), 72.7 (C-12), 22.3 (C-13), 20.1 (C-14), 16.5 (C-15), 109.6 (C-1'), 26.4 (C-2'), 27.3 (C-3') (9).

Compound 3 (solajiangxin $\mathrm{H}, \mathrm{C}_{18} \mathrm{H}_{28} \mathrm{O}_{4}$ ) presented as a colorless viscous oil; purity: 95.1\%; ${ }^{1} \mathrm{H}-\mathrm{NMR}(400 \mathrm{MHz}$,

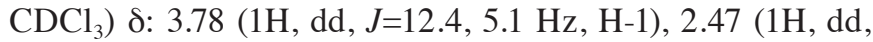
$J=16.1,12.3 \mathrm{~Hz}, \mathrm{Ha}-2), 2.57$ (1H, dd, $J=16.1,5.2 \mathrm{~Hz}, \mathrm{Hb}-2)$, 1.77 (1H, br t, $J=14.5 \mathrm{~Hz}, \mathrm{Ha}-6), 2.97$ (1H, br d, $J=14.5 \mathrm{~Hz}$, Hb-6), 1.52 (1H, m, H-7), 1.91 (1H, m, Ha-8), 1.43 (1H, m, Hb-8), 1.31 (1H, m, Ha-9), 2.07 (1H, m, Hb-9), $3.70(1 \mathrm{H}, \mathrm{d}$, $J=8.3$, Ha-12), 3.63 (1H, d, $J=8.3, \mathrm{Hb}-12), 1.31$ (3H, s, H-13),
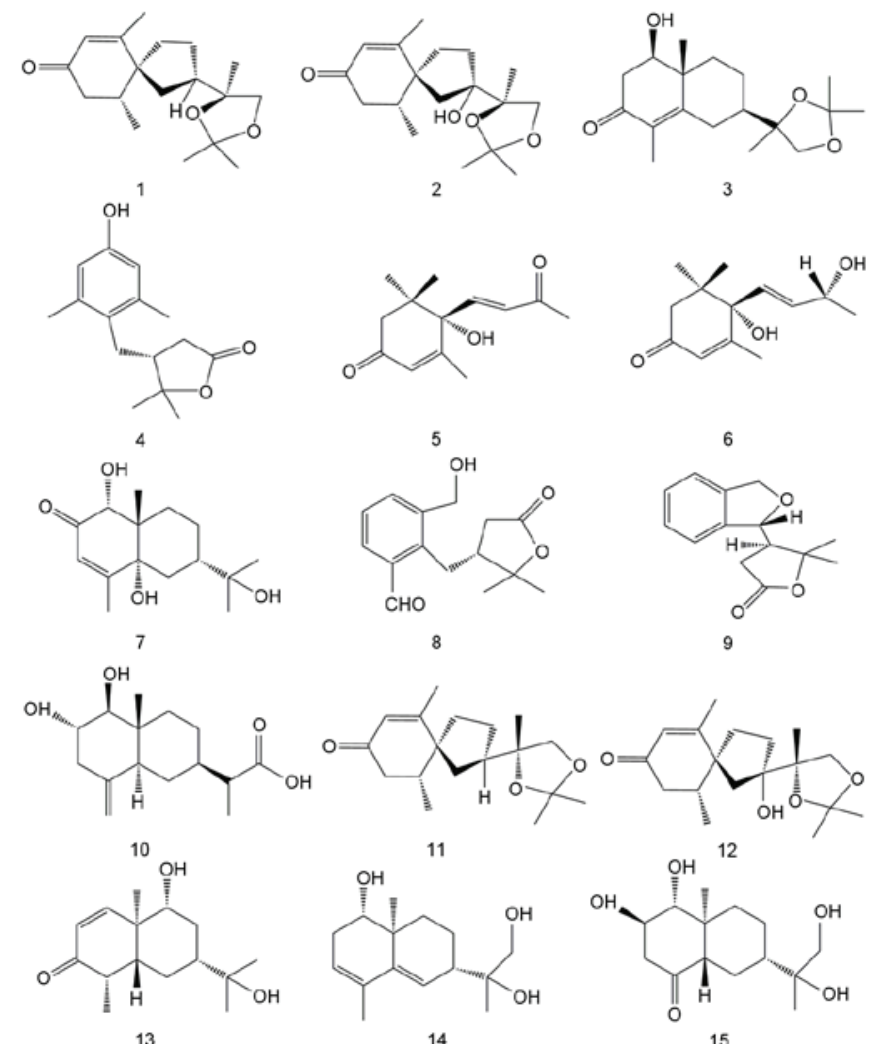

Figure 1. The chemical structure of 15 the sesquiterpenoids isolated from S. lyratum (compounds 1-15).

1.10 (3H, s, H-14), 1.68 (3H, s, H-15), 1.41 (3H, s, H-2'), 1.46

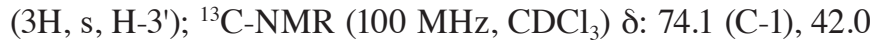
(C-2), 197.5 (C-3), 129.4 (C-4), 161.5 (C-5), 29.3 (C-6), 46.4 (C-7), 22.7 (C-8), 37.1 (C-9), 41.9 (C-10), 82.7 (C-11), 72.4 (C-12), 22.6 (C-13), 16.1 (C-14), 11.3 (C-15), 26.6 (C-2'), 27.3 (C-3') (9).

Compound 4 (lyratol D, $\mathrm{C}_{15} \mathrm{H}_{20} \mathrm{O}_{3}$ ) presented as a pale yellow lamellar crystal; purity: $98.2 \%$; ${ }^{1} \mathrm{H}-\mathrm{NMR}(400 \mathrm{MHz}$, $\mathrm{CDCl}_{3}$ ) $\delta: 2.31$ (1H, dd, $\left.J=18.3,3.6, \mathrm{Ha}-3\right), 2.43$ (1H, dd, $J=18.3$, 10.9, Hb-3), 2.54 (1H, m, H-4), 2.43 (1H, dd, J=14.1, 5.0, Нa-6), 2.79 (1H, dd, J=14.1, 11.3, Hb-6), 6.43 (1H, s, H-9), 6.43 (1H, s, H-11), 1.46 (3H, s, H-13), 1.59 (3H, s, H-14), 2.56 (3H, s, H-15),

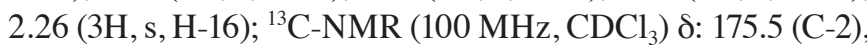
34.1 (C-3), 46.6 (C-4), 86.6 (C-5), 27.3 (C-6), 127.2 (C-7), 137.9 (C-8), 115.4 (C-9), 153.5 (C-10), 115.8 (C-11), 137.8 (C-12), 21.7 (C-13), 27.7 (C-14), 20.3 (C-15), 20.3 (C-16) (10).

Compound 5 (dehydrovomifoliol, $\mathrm{C}_{13} \mathrm{H}_{18} \mathrm{O}_{3}$ ) presented as a pale yellow oil; purity: $93.0 \%$; ${ }^{1} \mathrm{H}-\mathrm{NMR}\left(400 \mathrm{MHz}, \mathrm{CDCl}_{3}\right) \delta$ : 2.37 (1H, d, J=17.0 Hz, H-2a), 2.51 (1H, d, J=17.0 Hz, H-2b), $5.98(1 \mathrm{H}, \mathrm{s}, \mathrm{H}-4), 6.94(1 \mathrm{H}, \mathrm{d}, J=15.4 \mathrm{~Hz}, \mathrm{H}-7), 6.44(1 \mathrm{H}, \mathrm{d}$, $J=15.4 \mathrm{~Hz}, \mathrm{H}-8), 2.33$ (3H, s, H-10), 1.89 (3H, s, H-11), 1.08 (3H, s, H-12), 1.01 (3H, s, H-13); ${ }^{13} \mathrm{C}-\mathrm{NMR}\left(100 \mathrm{MHz}, \mathrm{CDCl}_{3}\right.$ ) ঠ: 41.6 (C-1), 49.3 (C-2), 197.3 (C-3), 127.8 (C-4), 160.1 (C-5), 79.5 (C-6), 144.8 (C-7), 130.5 (C-8), 196.8 (C-9), 28.2 (C-10), 18.7 (C-11), 24.5 (C-12), 22.9 (C-13) (11).

Compound 6 (blumenol $\mathrm{A}, \mathrm{C}_{13} \mathrm{H}_{20} \mathrm{O}_{3}$ ) presented as a pale

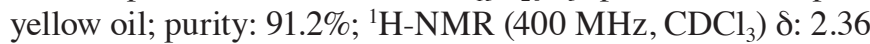
(1H, d, $J=17.3 \mathrm{~Hz}, \mathrm{H}-2 \mathrm{a}), 2.57$ (1H, d, J=17.3 Hz, H-2b), 5.98 (1H, s, H-4), 5.80 (1H, d, J=15.5 Hz, H-7), 5.81 (1H, dd, J=15.5, $5.3 \mathrm{~Hz}, \mathrm{H}-8), 4.58$ (1H, m, H-9), 1.32 (3H, d, J=6.7, H-10), 1.08 
(3H, s, H-11), 1.05 (3H, s, H-12), 1.85 (3H, s, H-13); ${ }^{13} \mathrm{C}-\mathrm{NMR}$

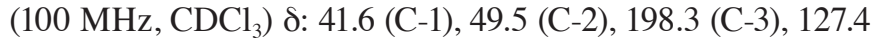
(C-4), 162.1 (C-5), 78.5 (C-6), 128.6 (C-7), 135.4 (C-8), 68.8 (C-9), 23.2 (C-10), 22.3 (C-11), 23.8 (C-12), 18.9 (C-13) (11).

Compound 7 (solajiangxin $\mathrm{A}, \mathrm{C}_{15} \mathrm{H}_{24} \mathrm{O}_{4}$ ) presented as a white powder; purity: $917.5 \%$; ${ }^{1} \mathrm{H}-\mathrm{NMR}\left(400 \mathrm{MHz}, \mathrm{DMSO}-d_{6}\right)$ ઈ: 3.30 (1H, br s, H-1), 5.58 (1H, s, H-3), 1.67 (1H, dd, J=13.1, $3.7 \mathrm{~Hz}, \mathrm{Ha}-6), 1.14$ (1H, dd, $J=13.1,6.2 \mathrm{~Hz}, \mathrm{Hb}-6), 1.78(1 \mathrm{H}$, m, H-7), 1.54 (1H, br d, J=12.6 Hz, Ha-8), 1.46 (1H, m, Hb-8), 2.17 (1H, br t, J=13.4 Hz, Ha-9), 1.18 (1H, m, Hb-9), 1.17 (3H, s, H-12), 1.19 (3H, s, H-13), 0.86 (3H, s, H-14), 1.90 (3H, s, H-15), 6.61 (1H, br s, C1-OH), $5.32(1 \mathrm{H}, \mathrm{s}, \mathrm{C} 5-\mathrm{OH}), 4.11$ $(1 \mathrm{H}, \mathrm{s}, \mathrm{C} 11-\mathrm{OH}) ;{ }^{13} \mathrm{C}-\mathrm{NMR}\left(100 \mathrm{MHz}^{\left.-\mathrm{CDCl}_{3}\right)}\right.$ ): $79.9(\mathrm{C}-1)$, 197.5 (C-2), 122.7 (C-3), 164.5 (C-4), 73.3 (C-5), 29.1 (C-6), 42.3 (C-7), 20.1 (C-8), 28.5 (C-9), 40.7 (C-10), 70.8 (C-11), 27.1 (C-12), 27.5 (C-13), 20.2 (C-14), 19.0 (C-15) (12).

Compound 8 (solajiangxin $\mathrm{B}, \mathrm{C}_{15} \mathrm{H}_{18} \mathrm{O}_{4}$ ) presented as a colorless viscous oil; purity: 90.6\%; ${ }^{1} \mathrm{H}-\mathrm{NMR}(400 \mathrm{MHz}$,

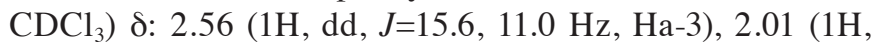
dd, $J=15.6,6.1 \mathrm{~Hz}, \mathrm{Hb}-3), 2.41$ (1H, m, H-4), 3.12 (2H, br d, $J=6.6 \mathrm{~Hz}, \mathrm{H}-6), 7.51$ (1H, br d, $J=7.7 \mathrm{~Hz}, \mathrm{H}-9), 7.31$ (1H, br t, $J=7.7 \mathrm{~Hz}, \mathrm{H}-10), 7.63$ (1H, br d, $J=7.6 \mathrm{~Hz}, \mathrm{H}-11), 1.39$ (3H, s, H-13), 1.54 (3H, s, H-14), 4.70 (2H, s, H-15), 10.19 (1H, s, H-16); ${ }^{13} \mathrm{C}-\mathrm{NMR}\left(100 \mathrm{MHz}, \mathrm{CDCl}_{3}\right)$ d: 175.6 (C-2), 33.6 (C-3), 46.7 (C-4), 87.4 (C-5), 26.3 (C-6), 139.2 (C-7), 134.1 (C-8), 136.5 (C-9), 127.9 (C-10), 134.1 (C-11), 140.3 (C-12), 21.7 (C-13), 26.5 (C-14), 62.0 (C-15), 193.6 (C-16) (12).

Compound 9 (solajiangxin $\mathrm{C}, \mathrm{C}_{15} \mathrm{H}_{18} \mathrm{O}_{3}$ ) presented as a colorless viscous oil; purity: 91.4\%; ${ }^{1} \mathrm{H}-\mathrm{NMR}\left(400 \mathrm{MHz}, \mathrm{CDCl}_{3}\right.$ )

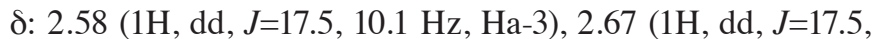
$9.0 \mathrm{~Hz}, \mathrm{Hb}-3), 3.17$ (1H, m, H-4), 5.88 (1H, d, J=9.2 Hz, H-6), 7.06 (1H, br d, J=7.6 Hz, H-9), 7.13 (1H, br t, $J=7.6 \mathrm{~Hz}, \mathrm{H}-10)$, 7.05 (1H, br d, J=7.6 Hz, H-11), 1.10 (3H, s, H-13), 1.35 (3H, s, H-14), 4.69 (1H, d, J=12.1 Hz, Ha-15), $4.82(1 \mathrm{H}, \mathrm{d}, J=12.1 \mathrm{~Hz}$, $\mathrm{Hb}-15), 2.47$ (3H, s, H-16); ${ }^{13} \mathrm{C}-\mathrm{NMR}\left(100 \mathrm{MHz}, \mathrm{CDCl}_{3}\right) \delta$ : 176.3 (C-2), 32.5 (C-3), 51.4 (C-4), 70.4 (C-5), 79.4 (C-6), 135.0 (C-7), 137.6 (C-8), 132.8 (C-9), 128.7 (C-10), 129.3 (C-11), 138.4 (C-12), 26.8 (C-13), 29.0 (C-14), 64.3 (C-15), 21.0 (C-16) (12).

Compound 10 (solajiangxin $\mathrm{D}, \mathrm{C}_{15} \mathrm{H}_{24} \mathrm{O}_{4}$ ) presented as a white powder; purity: $90.2 \%$; ${ }^{1} \mathrm{H}-\mathrm{NMR}\left(400 \mathrm{MHz}, \mathrm{DMSO}-d_{6}\right)$

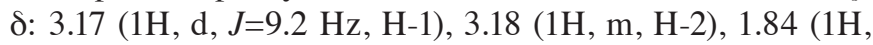
br t, $J=12.5 \mathrm{~Hz}, \mathrm{Ha}-3), 2.30(1 \mathrm{H}, \mathrm{dd}, J=12.5,5.1 \mathrm{~Hz}, \mathrm{Hb}-3)$, 1.53 (1H, br d, $J=11.4 \mathrm{~Hz}, \mathrm{H}-5), 1.18$ (1H, m, Ha-6), $1.51(1 \mathrm{H}$, m, Hb-6), 2.01 (1H, m, H-7), 1.17 (1H, m, Ha-8), 1.38 (1H, m, Hb-8), 1.74 (1H, m, Ha-9), 1.26 (1H, m, Hb-9), 1.41 (1H, m, H-11), 1.12 (3H, d, J=7.1 Hz, H-13), 0.49 (3H, s, H-14), 4.30 (1H, s, Ha-15), 4.70 (1H, s, Hb-15), $5.81(1 \mathrm{H}$, br s, C1-OH), $4.80(1 \mathrm{H}$, br s, C2-OH), $11.35\left(1 \mathrm{H}\right.$, br s, COOH); ${ }^{13} \mathrm{C}-\mathrm{NMR}(100 \mathrm{MHz}$, DMSO- $\left.d_{6}\right)$ d: 84.3 (C-1), 69.8 (C-2), 43.4 (C-3), 147.5 (C-4), 47.1 (C-5), 27.4 (C-6), 44.2 (C-7), 23.3 (C-8), 36.2 (C-9), 39.1 (C-10), 39.5 (C-11), 177.4 (C-12), 13.9 (C-13), 11.3 (C-14), 107.5 (C-15) (13).

Compound 11 (solajiangxin $\mathrm{E}, \mathrm{C}_{18} \mathrm{H}_{28} \mathrm{O}_{3}$ ) presented as a colorless viscous oil; purity: 94.0\%; ${ }^{1} \mathrm{H}-\mathrm{NMR}(400 \mathrm{MHz}$,

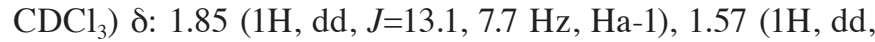
$J=13.1,11.4$ Hz, Hb-1), 2.04 (1H, m, H-2), 1.76 (1H, m, Ha-3), 1.60 (1H, m, Hb-3), 1.61 (1H, m, Ha-4), 1.78 (1H, m, Hb-4), 5.67 (1H, s, H-7), 2.43 (1H, dd, J=16.6, 4.8 Hz, Ha-9), 2.08 (1H, dd, J=16.6, 4.2 Hz, Hb-9), 1.93 (1H, m, H-10), $3.74(1 \mathrm{H}$, d, $J=8.3 \mathrm{~Hz}, \mathrm{Ha}-12), 3.84(1 \mathrm{H}, \mathrm{d}, J=8.3 \mathrm{~Hz}, \mathrm{Hb}-12), 1.32(3 \mathrm{H}$, s, H-13), 1.94 (3H, s, H-14), 1.00 (3H, d, J=7.1 Hz, H-15), 1.41 (3H, s, H-2'), 1.40 (3H, s, H-3'); ${ }^{13} \mathrm{C}-\mathrm{NMR}\left(100 \mathrm{MHz}, \mathrm{CDCl}_{3}\right.$ ) ઈ: 37.1 (C-1), 47.3 (C-2), 28.6 (C-3), 34.1 (C-4), 50.2 (C-5), 166.0 (C-6), 125.8 (C-7), 199.3 (C-8), 43.1 (C-9), 38.6 (C-10), 82.1 (C-11), 73.2 (C-12), 24.8 (C-13), 20.7 (C-14), 15.9 (C-15), 109.1 (C-1'), 26.8 (C-2'), 27.0 (C-3') (13).

Compound 12 (2-hydroxysolajiangxin E, $\mathrm{C}_{18} \mathrm{H}_{28} \mathrm{O}_{4}$ ) presented as a colorless viscous oil; purity: 92.3\%; ${ }^{1} \mathrm{H}-\mathrm{NMR}$

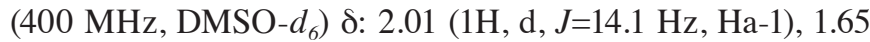
(1H, d, J=14.1 Hz, Hb-1), 1.94 (1H, m, Ha-3), 1.55 (1H, m, Hb-3), 1.80 (1H, m, Ha-4), 1.76 (1H, m, Hb-4), 5.68 (1H, s, H-7), 2.48 (1H, dd, $J=16.3,5.0 \mathrm{~Hz}, \mathrm{Ha}-9), 2.13(1 \mathrm{H}, \mathrm{dd}, J=16.3,4.1 \mathrm{~Hz}$, Hb-9), 2.51 (1H, m, H-10), 3.54 (1H, d, J=8.7 Hz, Ha-12), 4.07 (1H, d, J=8.7 Hz, Hb-12), 1.18 (3H, s, H-13), 1.83 (3H, s, H-14), 0.81 (3H, d, J=7.0 Hz, H-15), 1.23 (3H, s, H-2'), 1.35 (3H, s, H-3'), 4.49 (1H, s, C1-OH); ${ }^{13} \mathrm{C}-\mathrm{NMR}\left(100 \mathrm{MHz}, \mathrm{DMSO}-d_{6}\right) \mathrm{\delta}$ : 44.3 (C-1), 84.2 (C-2), 35.0 (C-3), 33.8 (C-4), 49.2 (C-5), 166.1 (C-6), 124.7 (C-7), 198.5 (C-8), 43.6 (C-9), 39.4 (C-10), 84.6 (C-11), 72.1 (C-12), 22.8 (C-13), 20.6 (C-14), 15.9 (C-15), 108.4 (C-1'), 27.0 (C-2'), 27.3 (C-3') (13).

Compound 13 (lyratol A, $\mathrm{C}_{15} \mathrm{H}_{24} \mathrm{O}_{3}$ ) presented as a colorless viscous oil; purity: 95.1\%; ${ }^{1} \mathrm{H}-\mathrm{NMR}\left(400 \mathrm{MHz}, \mathrm{CDCl}_{3}\right.$ ) ঠ: $7.17(1 \mathrm{H}, \mathrm{d}, J=9.6 \mathrm{~Hz}, \mathrm{H}-1), 5.70(1 \mathrm{H}, \mathrm{d}, J=9.6, \mathrm{H}-2), 2.54$ (1H, m, H-4), 1.39 (1H, m, H-5), 1.26 (1H, m, Ha-6), $1.70(1 \mathrm{H}$, m, Hb-6), 1.45 (1H, m, H-7), 1.91 (1H, m, Ha-8), 1.33 (1H, m, Hb-8), 3.27 (1H, dd, J=11.0, 4.5 Hz, H-9), 1.12 (3H, s, H-12), 1.20 (3H, s, H-13), 1.05 (3H, d, J=6.8 Hz, H-14), $0.96(3 \mathrm{H}$,

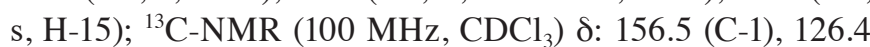
(C-2), 201.5 (C-3), 41.5 (C-4), 46.8 (C-5), 23.1 (C-6), 46.6 (C-7), 31.0 (C-8), 74.5 (C-9), 41.4 (C-10), 72.1 (C-11), 27.0 (C-12), 26.9 (C-13), 12.5 (C-14), 11.2 (C-15) (14).

Compound 14 (lyratol $\mathrm{B}, \mathrm{C}_{15} \mathrm{H}_{24} \mathrm{O}_{3}$ ) presented as a colorless viscous oil; purity: $94.6 \%$; ${ }^{1} \mathrm{H}-\mathrm{NMR}\left(400 \mathrm{MHz} \mathrm{CDCl}_{3}\right) \delta: 3.39$ (1H, dd, J=11.2, 4.3 Hz, H-1), 2.26 (1H, m, Ha-2), 2.27 (1H, m, Hb-2), 5.14 (1H, br d, J=4.6 Hz, H-3), 5.34 (1H, br s, H-6), 2.25 (1H, m, H-7), 1.92 (1H, m, Ha-8), 1.44 (1H, m, Hb-8), 1.12 (1H, m, Ha-9), 2.04 (1H, m, Hb-9), 3.49 (1H, d, J=11.0 Hz, Ha-12), 3.58 (1H, d, J=11.0 Hz, Ha-12), 1.08 (3H, s, H-13), 1.89 (3H, s, H-14), 0.99 (3H, s, H-15); ${ }^{13} \mathrm{C}-\mathrm{NMR}\left(100 \mathrm{MHz}, \mathrm{CDCl}_{3}\right.$ )

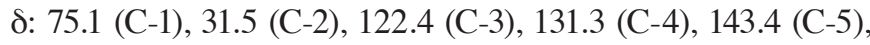
121.1 (C-6), 43.8 (C-7), 19.0 (C-8), 33.8 (C-9), 37.5 (C-10), 74.6 (C-11), 67.3 (C-12), 21.4 (C-13), 19.6 (C-14), 16.3 (C-15) (14).

Compound 15 (lyratol C, $\mathrm{C}_{15} \mathrm{H}_{26} \mathrm{O}_{4}$ ) presented as a colorless prismatic crystal; purity: $92.5 \%$; ${ }^{1} \mathrm{H}-\mathrm{NMR}\left(400 \mathrm{MHz}, \mathrm{C}_{5} \mathrm{D}_{5} \mathrm{~N}\right)$

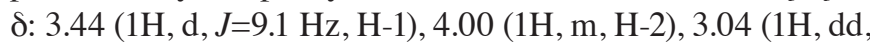
$J=12.4,5.5 \mathrm{~Hz}, \mathrm{Ha}-3), 2.55(1 \mathrm{H}, \mathrm{dd}, J=12.4,11.7 \mathrm{~Hz}, \mathrm{Hb}-3)$, 1.83 (1H, dd, J=10.3, 3.6 Hz, H-5), 1.64 (1H, m, Ha-6), 1.88 (1H, m, Hb-6), 2.21 (1H, m, H-7), 1.53 (1H, m, Ha-8), $2.04(1 \mathrm{H}$, m, Hb-8), 2.73 (1H, m, Ha-9), 1.24 (1H, m, Ha-9), 4.10 (1H, d, $J=10.8 \mathrm{~Hz}, \mathrm{Ha}-12), 3.83$ (1H, d, $J=10.8 \mathrm{~Hz}, \mathrm{Hb}-12), 1.50(3 \mathrm{H}$, s, H-13), 1.28 (3H, s, H-14), 4.83 (1H, s, Ha-15), 4.94 (1H, s,

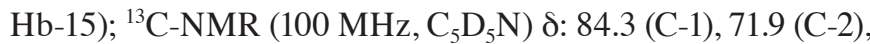
44.0 (C-3), 148.5 (C-4), 48.9 (C-5), 25.0 (C-6), 45.6 (C-7), 21.8 (C-8), 38.7 (C-9), 39.8 (C-10), 74.6 (C-11), 68.7 (C-12), 21.6 (C-13), 11.5 (C-14), 107.6 (C-15) (2).

Cytotoxic potential of sesquiterpenoids from S. lyratum. The anti-proliferation effect of sesquiterpenoids on human breast adenocarcinoma MCF-7, human intestinal adenocarcinoma HCT-8, human lung carcinoma A-549, human gastric cancer 
Table I. Cytotoxicity of 15 sesquiterpenoids against cultured MCF-7, HCT-8, A-549, SGC-7901 and BEL-7402 cells.

\begin{tabular}{|c|c|c|c|c|c|c|}
\hline \multirow[b]{2}{*}{ No. } & \multirow[b]{2}{*}{ Compound } & \multicolumn{5}{|c|}{$\mathrm{IC}_{50}(\mu \mathrm{g} / \mathrm{ml})$} \\
\hline & & MCF-7 & HCT-8 & A-549 & SGC-7901 & BEL-7402 \\
\hline 1 & Solajiangxin I & $18.9 \pm 0.8$ & $23.8 \pm 1.3$ & $17.6 \pm 0.7$ & $18.6 \pm 0.7$ & $23.5 \pm 3.1$ \\
\hline 2 & 7-Hydroxylsolajiangxin I & $43.1 \pm 1.3$ & $21.3 \pm 1.4$ & $16.4 \pm 1.0$ & $19.3 \pm 1.1$ & $32.1 \pm 0.9$ \\
\hline 3 & Solajiangxin H & $15.4 \pm 0.9$ & $19.6 \pm 2.3$ & $28.3 \pm 0.5$ & $4.8 \pm 0.3$ & $18.4 \pm 1.4$ \\
\hline 4 & Lyratol D & $33.6 \pm 1.8$ & $18.5 \pm 0.7$ & $19.3 \pm 0.8$ & $5.9 \pm 0.2$ & $41.5 \pm 0.8$ \\
\hline 5 & Dehydrovomifoliol & $19.3 \pm 2.0$ & $22.4 \pm 1.0$ & $20.3 \pm 1.2$ & $10.5 \pm 1.4$ & $51.0 \pm 1.2$ \\
\hline 6 & Blumenol A & $56.4 \pm 0.7$ & $16.5 \pm 4.1$ & $28.2 \pm 1.1$ & $13.5 \pm 1.6$ & $25.4 \pm 0.5$ \\
\hline 7 & Solajiangxin A & $38.9 \pm 1.5$ & $40.1 \pm 5.4$ & $30.3 \pm 1.8$ & $18.9 \pm 2.4$ & $41.2 \pm 1.3$ \\
\hline 8 & Solajiangxin B & $28.6 \pm 2.9$ & $23.6 \pm 2.6$ & $18.2 \pm 0.9$ & $21.3 \pm 2.5$ & $32.5 \pm 2.4$ \\
\hline 9 & Solajiangxin C & $35.1 \pm 1.5$ & $27.3 \pm 3.8$ & $25.8 \pm 2.1$ & $18.4 \pm 3.0$ & $12.5 \pm 0.4$ \\
\hline 10 & Solajiangxin D & $23.4 \pm 1.6$ & $18.6 \pm 1.1$ & $29.2 \pm 0.9$ & $14.1 \pm 1.5$ & $31.4 \pm 1.7$ \\
\hline 11 & Solajiangxin E & $31.2 \pm 2.3$ & $25.7 \pm 2.4$ & $17.8 \pm 1.1$ & $15.3 \pm 2.6$ & $28.9 \pm 0.7$ \\
\hline 12 & 2-Hydroxysolajiangxin E & $26.3 \pm 0.7$ & $18.4 \pm 2.9$ & $16.9 \pm 1.6$ & $12.4 \pm 1.8$ & $25.4 \pm 1.6$ \\
\hline 13 & Lyratol A & $31.5 \pm 1.4$ & $18.4 \pm 0.9$ & $11.3 \pm 0.5$ & $9.6 \pm 1.4$ & $25.7 \pm 2.4$ \\
\hline 14 & Lyratol B & $42.8 \pm 1.8$ & $15.1 \pm 1.2$ & $12.4 \pm 0.8$ & $8.7 \pm 1.0$ & $18.9 \pm 3.1$ \\
\hline 15 & Lyratol C & $35.8 \pm 2.1$ & $23.4 \pm 1.5$ & $13.6 \pm 3.2$ & $11.3 \pm 2.3$ & $31.2 \pm 0.8$ \\
\hline
\end{tabular}

Data are presented as the mean \pm the standard deviation. $\mathrm{IC}_{50}$, half maximal inhibitory concentration.
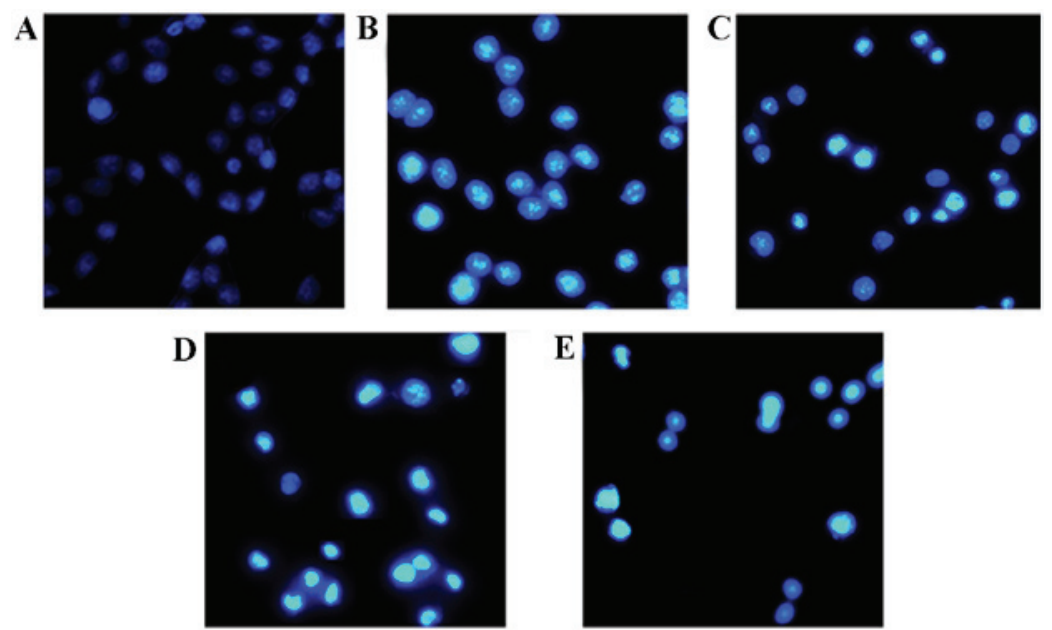

Figure 2. Effects of solajiangxin H and lyratol D on apoptosis in SGC-7901 cells by DAPI staining. SGC-7901 cells were treated with SOG and lyratol D at doses of 0 (control), 2 and $4 \mu \mathrm{g} / \mathrm{ml}$ for $48 \mathrm{~h}$, and then stained by DAPI and images were captured using a fluorescence microscope (magnification, x200). (A) Control; (B) $2 \mu \mathrm{g} / \mathrm{ml} \mathrm{SOG;} \mathrm{(C)} 2 \mu \mathrm{g} / \mathrm{ml}$ lyratol D; (D) $4 \mu \mathrm{g} / \mathrm{ml} \mathrm{SOG;} \mathrm{and} \mathrm{(E)} 4 \mu \mathrm{g} / \mathrm{ml} \mathrm{lyratol} \mathrm{D.} \mathrm{SOG,} \mathrm{solajiangxin} \mathrm{H.}$

SGC-7901 and human hepatocarcinoma BEL-7402 cells were studied using a CCK-8 assay, which has commonly been used to investigate the cytotoxicity of compounds (15). The results are presented in Table I. The $\mathrm{IC}_{50}$ values of the 15 sesquiterpenoids identified were between 4.8 and $56.4 \mu \mathrm{g} / \mathrm{ml}$. Compounds 3 (solajiangxin $\mathrm{H}$ ) and 4 (lyratol D) had high cytotoxicity against SGC-7901 cells ( $\mathrm{IC}_{50}=4.8$ and $5.9 \mu \mathrm{g} / \mathrm{ml}$, respectively), so they were selected for additional study into their anticancer mechanisms by DAPI staining and western blot analysis.

Effect of solajiangxin $H$ and lyratol $D$ on apoptosis in $S G C-7901$ cells. DAPI staining (16) was used to study whether the cytotoxic effects of solajiangxin $\mathrm{H}$ and lyratol $\mathrm{D}$ on
SGC-7901 cells were associated with apoptosis. As depicted in Fig. 2, subsequent to treatment with solajiangxin $\mathrm{H}$ and lyratol $\mathrm{D}$ at doses of 0 (control), 2 and $4 \mu \mathrm{g} / \mathrm{ml}$ for $48 \mathrm{~h}$, respectively, evident condensation of the nucleus of the SGC-7901 cells was induced, which can be regarded as a characteristic marker of apoptosis. Therefore, the cytotoxic effects of solajiangxin $\mathrm{H}$ and lyratol D on SGC-7901 cells were associated with apoptosis.

Effect of solajiangxin $H$ and lyratol $D$ on apoptotic proteins in SGC-7901 cells. A western blot assay was used to study the mechanism of apoptosis induced by solajiangxin $\mathrm{H}$ and lyratol D. The present study examined the expression changes 

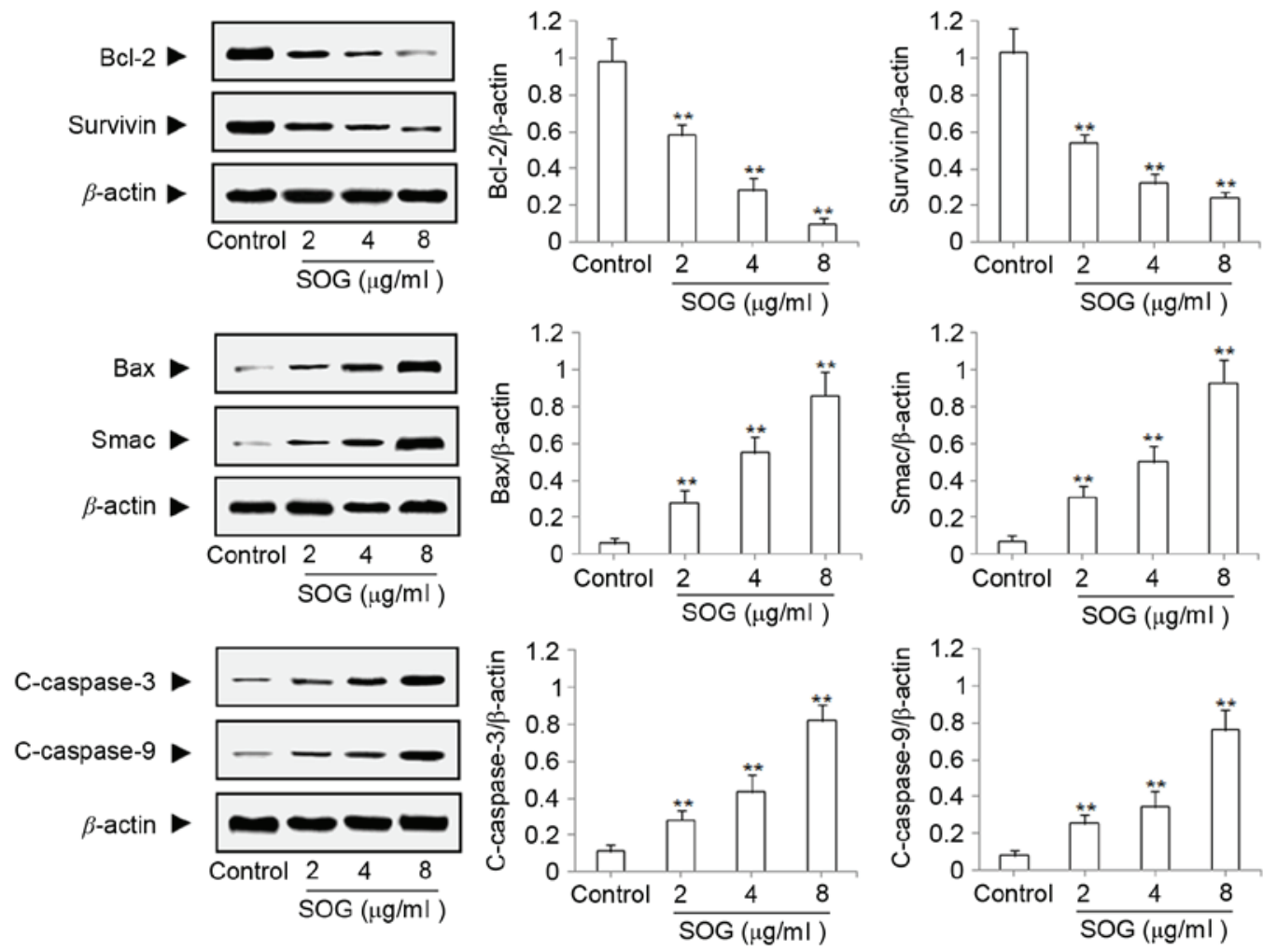

Figure 3. Effects of SOG on the antiapoptotic proteins Bcl-2 and survivin and the proapoptotic proteins Bax, Smac, c-caspase-3 and c-caspase-9 in SGC-7901 cells. SGC-7901 cells were treated with SOG at concentrations of 0 (control), 2, 4 and $8 \mu \mathrm{g} / \mathrm{ml}$ for $48 \mathrm{~h}$, and their total proteins were analyzed by western blot assay. ${ }^{* *} \mathrm{P}<0.01$, compared with control. SOG, solajiangxin H; Bcl-2, B-cell lymphoma 2; Bax, Bcl-2-like protein 4; Smac, second mitochondria-derived activator of caspase; c-caspase, cleaved caspase.
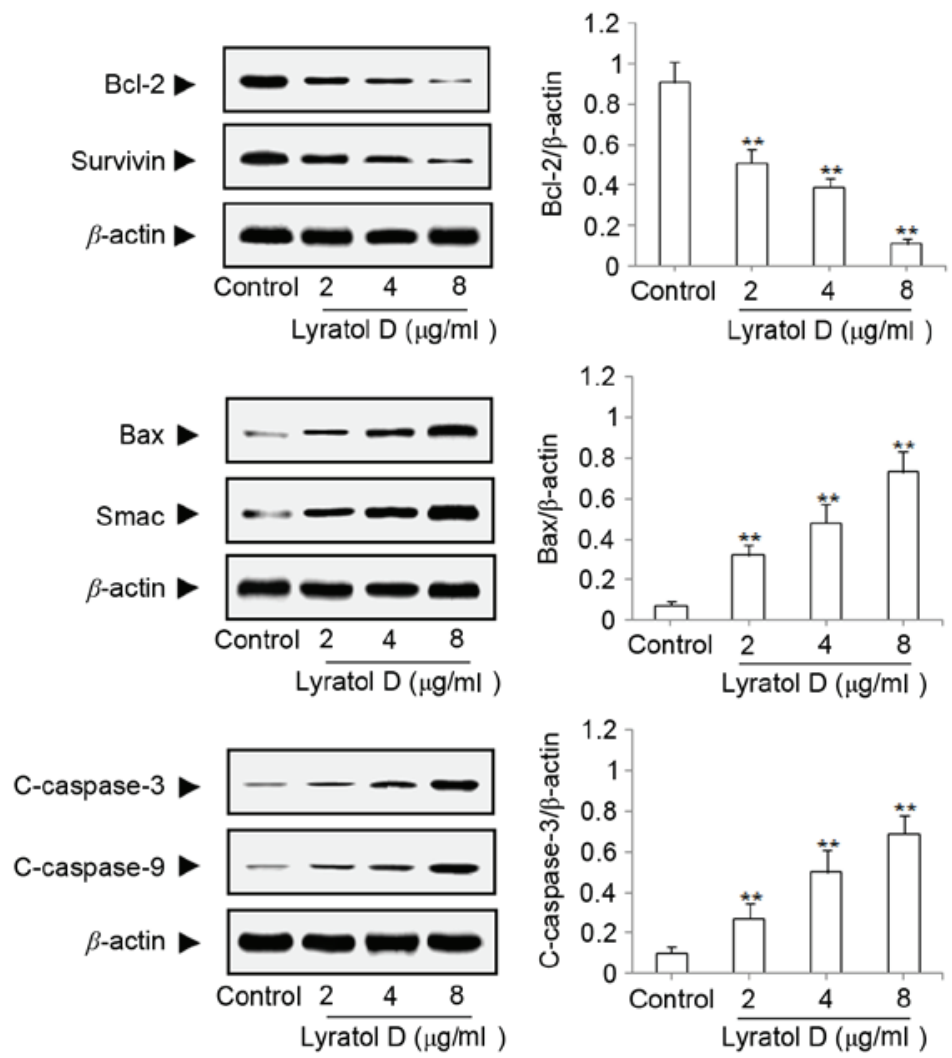
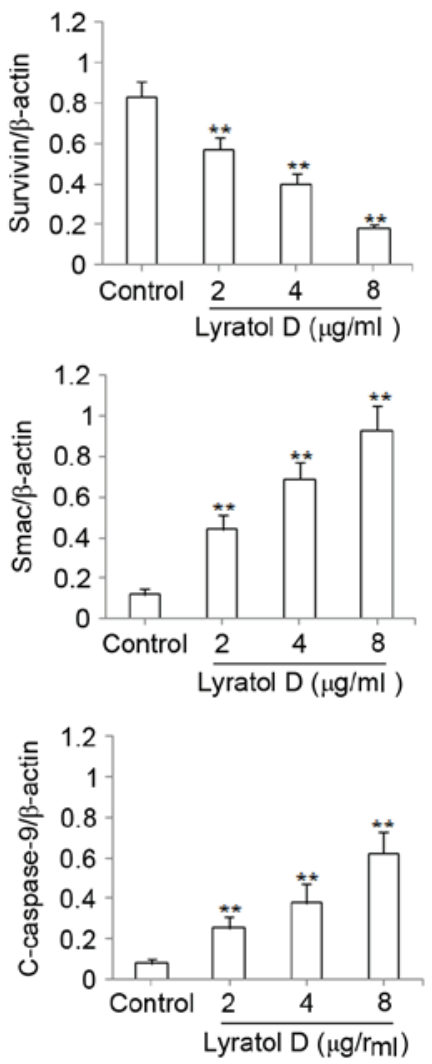

Figure 4. Effects of lyratol D on the antiapoptotic proteins Bcl-2 and survivin, and the proapoptotic proteins Bax, Smac, c-caspase-3 and c-caspase-9 in SGC-7901 cells. The SGC-7901 cells were treated with lyratol D at concentrations of 0 (control), 2,4 and $8 \mu \mathrm{g} / \mathrm{ml}$ for $48 \mathrm{~h}$, respectively, and total protein content was analyzed by western blot assay. ${ }^{* *} \mathrm{P}<0.01$, compared with control. Bcl-2, B-cell lymphoma 2; Bax, Bcl-2-like protein 4; Smac, second mitochondria-derived activator of caspase; c-caspase, cleaved caspase. 
of apoptotic proteins, including Bcl-2, survivin, Bax, Smac, c-caspase-3 and c-caspase-9 subsequent to treatment with solajiangxin $\mathrm{H}$ or lyratol D at concentrations of 2, 4 or $8 \mu \mathrm{g} / \mathrm{ml}$ for $48 \mathrm{~h}$, respectively. As Figs. 3 and 4 demonstrate, the results indicated that solajiangxin $\mathrm{H}$ and lyratol D may significantly $(\mathrm{P}<0.01)$ downregulate the expression of the antiapoptotic proteins $\mathrm{Bcl}-2$ and survivin, and upregulate the expression of the proapoptotic proteins Bax, Smac, c-caspase-3 and c-caspase-9 $(\mathrm{P}<0.01)$, in a dose-dependent manner $(17)$.

\section{Discussion}

In the present study, 15 sesquiterpenoids were isolated from S. lyratum, and their antiproliferation effects on MCF-7, HCT-8, A-549, SGC-7901 and BEL-7402 cell lines were investigated using a CCK-8 assay. According to the results of CCK-8 assay, solajiangxin $\mathrm{H}$ and lyratol $\mathrm{D}$, which had strong cytotoxicity against SGC-7901 cells $\left(\mathrm{IC}_{50}=4.8\right.$ and $5.9 \mu \mathrm{g} / \mathrm{ml}$, respectively), were selected to study their anticancer mechanisms by DAPI staining and western blot assay. These techniques demonstrated that the cytotoxicity of solajiangxin $\mathrm{H}$ and lyratol D against SGC-7901 cells was associated with apoptosis.

Previous studies revealed that apoptotic proteins, including Bcl-2, survivin, Bax, Smac, caspase-3 and caspase-9, serve important roles in mitochondria-mediated apoptosis $(17,18)$. The associations between the proteins are complex. For example, the antiapoptotic Bcl-2 protein can suppress the release of the proapoptotic Smac protein from the mitochondria to the cytoplasm, but the proapoptotic Bax protein can inhibit the activity of Bcl-2 protein $(19,20)$. The active form of caspase-3, c-caspase-3, is an important signal for apoptosis, and the activation of caspase- 3 is associated with the activation of caspase-9 into c-caspase-9 (21). The antiapoptotic survivin protein can inhibit the c-caspase- 9 to activate caspases-3, but Smac can inhibit the activity of survivin (22). Thus, changes in expression level and activity of the apoptotic proteins Bcl-2, survivin, Bax, Smac, caspase- 3 and caspase- 9 are directly associated with apoptosis. In the present study, changes in the expression levels of apoptotic proteins, including Bcl-2, survivin, Bax, Smac, c-caspase-3 and c-caspase-9, subsequent to treatment with solajiangxin $\mathrm{H}$ and lyratol $\mathrm{D}$ was investigated. The results indicated that solajiangxin $\mathrm{H}$ and lyratol $\mathrm{D}$ significantly downregulates the expression of the antiapoptotic proteins Bcl-2 and survivin, and upregulates the expression levels of the proapoptotic proteins Bax, Smac, c-caspase-3 and c-caspase-9 in dose-dependent manners.

In conclusion, the present study demonstrated that 15 sesquiterpenoids isolated from $S$. lyratum exhibit cytotoxicity against MCF-7, HCT-8, A-549, SGC-7901 and BEL-7402 cells. The results of studies on the anticancer mechanisms of solajiangxin $\mathrm{H}$ and lyratol D in SGC-7901 cells indicated that the anticancer mechanisms may be associated with mitochondria-mediated apoptosis. However, additional studies on mechanisms of these sesquiterpenoids are required as they may be candidates for treatment of cancer.

\section{Acknowledgements}

The present study was supported by the Foundation of the Priority Academic Program Development of Jiangsu Higher
Education Institutions and the National Natural Science Foundation of China (grant no. 81202954).

\section{References}

1. Sun LX, Bi KS and Wang MW: Progress in the studies of Solanum lyratum Thunb. J Shenyang Pharm Univ 23: 251-255, 2006.

2. Ren Y, Zhang DW and Dai SJ: Chemical constituents from Solanum lyratum. Chin J Nat Med 7: 203-205, 2009.

3. Yang L, Feng F and Gao Y: Chemical constituents from herb of Solanum lyratum. Zhongguo Zhong Yao Za Zhi 34: 1805-1808, 2009 (In Chinese).

4. Wan FS, Wu J, Li H, Tu S and Yu LH: Study on apoptosis of human stomach SGC-7901 cells induced by extracts of Solanum lyratum. Zhong Yao Cai 32: 245-249, 2009 (In Chinese).

5. Yu LH, Xu BH, Wu J, Li H, Tu S and Wan FS: Influence of Solanum lyratum Thunb etract on apoptosis and the expression of bcl-xl/bid genes in human stomach cancer SGC-7901 cells. Chin Tradit Pat Med 30: 1744-1748, 2008.

6. Hsu SC, Lu JH, Kuo CL, Yang JS, Lin MW, Chen GW, Su CC, Lu HF and Chung JG: Crude extracts of Solanum lyratum induced cytotoxicity and apoptosis in a human colon adenocarcinoma cell line (Colo 205). Anticancer Res 28: 1045-1054, 2008.

7. Ren J, Feng GN, Wang MW and Sun LX: The primary study on the anti-tumor effect of tatal saponin of Solanum lyratum Thunb. Cance Res Prevent Treat 33: 262-264, 2006 (In Chinese).

8. Sun LX, Ren J, Wang MW and Bi KS: Preliminary study on the anti-tumor effect of the extracts of Solanum lyratum Thunb. J Shenyang Pharm Univ 22: 210-212, 2005.

9. Li GS, Yao F, Zhang L, Yue XD and Dai SJ: New sesquiterpenoids derivatives from Solanum lyratum and their cytotoxicies. J Asian Nat Prod Res 16: 129-134, 2014.

10. Ren Y, Shen L, Zhang DW and Dai SJ: Two new sesquiterpenoids from Solanum lyratum with cytotoxic activities. Chem Pharm Bull (Tokyo) 57: 408-410, 2009.

11. Yue XD, Yue XD, Yao F, Zhang L, Li GS and Dai SJ: Sesquiterpenoids from Solanum lyratum. Zhongguo Zhong Yao Za Zhi 39: 453-456, 2014 (In Chinese).

12. Yao F, Song QL, Zhang L, Li GS and Dai SJ: Solajiangxins A-C, three new cytotoxic sesquiterpenoids from Solanum lyratum. Fitoterapia 89: 200-204, 2013.

13. Yao F, Song QL, Zhang L, Li GS and Dai SJ: Three new cytotoxic sesquiterpenoids from Solanum lyratum. Phytochem Lett 6: 453-456, 2013.

14. Dai SJ, Shen L and Ren Y: Two new eudesmane-type sesquiterpenoids from Olanum lyratum. Nat Prod Res 23: 1196-1200,2009.

15. Xie B, Zhou JP, Shu GS, Liu DC, Zhou J, Chen J and Yuan L: Restoration of klotho gene expression induces apoptosis and autophagy in gastric cancer cells: Tumor suppressive role of klotho in gastric cancer. Cancer Cell Int 13: 18, 2013.

16. Baharara J, Namvar F, Ramezani T, Mousavi M and Mohamad R: Silver nanoparticles biosynthesized using Achillea biebersteinii flower extract: Apoptosis induction in MCF-7 cells via caspase activation and regulation of $\mathrm{Bax}$ and $\mathrm{Bcl}-2$ gene expression. Molecules 20: 2693-2706, 2015.

17. Peng W, Wu JG, Jiang YB, Liu YJ, Sun T, Wu N and Wu CJ: Antitumor activity of 4-O-(2'-O-acetyl-6'-O-p-coumaroyl- $\beta$-Dglucopyranosyl)-p-coumaric acid against lung cancers via mitochondrial-mediated apoptosis. Chem Biol Interact 233: 8-13, 2015.

18. Shi YG: A structural view of mitochondria-mediated apoptosis. Nat Struct Biol 8: 394-401, 2001.

19. Deng YB, Lin YH and Wu XW: TRAIL-induced apoptosis requires Bax-dependent mitochondrial release of Smac/DIABLO. Genes Dev 16: 33-45, 2002.

20. Du C, Fang M, Li Y, Li L and Wang X: Smac, a mitochondrial protein that promotes cytochrome c-dependent caspase activation by eliminating IAP inhibition. Cell 102: 33-42, 2000.

21. Zhang XJ, Mei WL, Tan GH, Wang CC, Zhou SL, Huang FR, Chen B, Dai HF and Huang FY: Strophalloside induces apoptosis of SGC-7901 cells through the mitochondrion-dependent caspase-3 pathway. Molecules 20: 5741-5728, 2015.

22. Ambrosini G, Adida C and Altieri DC: A novel anti-apoptosis gene, surviving, expressed in cancer and lymphoma. Nat Med 3: 917-921, 1997. 